\title{
Tests of ChPT in Primakoff reactions at COMPASS
}

\author{
Stefanie Grabmüller*t for the COMPASS collaboration \\ Technische Universität München \\ E-mail: stefanie.grabmueller@tum.de
}

\begin{abstract}
Chiral Perturbation Theory effectively describes QCD at low energies, expressing the dynamics in terms of $m_{\pi}, f_{\pi}$ and higher-order low-energy constants. These have been adjusted to experimental results, e.g. on pion-pion scattering, and lead to predictions of quantities such as the pion polarizabilities or 2-pion production cross-sections.

The COMPASS experiment at CERN [1] is a unique tool for measurements of these quantities in pion-photon reactions, occurring in the Coulomb field of heavy nuclei with a high-energetic pion beam. The status of the polarizability determination via $\pi^{-} \gamma \rightarrow \pi^{-} \gamma$ is presented. The second focus is put on the measurement of the cross-section $\pi^{-} \gamma \rightarrow 3 \pi$ at low center-of-mass energies, extracted through partial-wave analysis methods [2].
\end{abstract}

Sixth International Conference on Quarks and Nuclear Physics,

April 16-20, 2012

Ecole Polytechnique, Palaiseau, Paris

\footnotetext{
* Speaker.

$\dagger$ This work is supported by the German BMBF, the Maier-Leibnitz-Labor München, the DFG Cluster of Excellence Exc153, and CERN-RFBR grant 08-02-91009.
} 


\section{Primakoff Reactions at COMPASS}

In the framework of Chiral Perturbation Theory (ChPT), pions are the Goldstone bosons of the spontaneous breaking of chiral symmetry. ChPT provides an effective theory for the strong interaction, described by Quantum ChromoDynamics (QCD), at low energies. It is based on scattering of bound systems (with pions and nucleons as the degrees of freedom) and effective couplings at their scattering vertices. The inner quark-gluon dynamics are condensed into low-energy constants, so that the scattering dynamics is expressed as expansion in the particle momenta with parameters determined by $m_{\pi}, f_{\pi}$ in leading order (tree diagrams), additional constants $\ell_{i}, i=1 \ldots 6$, for the nextorder calculations (loop diagrams), and more constants for higher-order calculations. In addition, ChPT calculations can consistently include interactions described by Quantum ElectroDynamics (QED), like $\pi \gamma$ scattering.

Properties of pions, and their interactions, are an important input to confirm that ChPT is the correct representation of QCD at low energies, in addition to established results like the pion scattering lengths obtained in kaon decay experiments. Of great interest in this view are $\pi \gamma$ reactions, which are experimentally accessible via the Primakoff effect: When high-energetic particles, e.g. pions, impinge on nuclear targets, a significant fraction of them scatters off the field quanta of the electro-magnetic field surrounding the heavy nucleus, i.e. quasi-real photons. In experimental data, photon exchange is apparent in the region of very low momentum transfer $t^{\prime} \lesssim 0.001 \mathrm{GeV}^{2} / c^{2}$, with $t^{\prime}=|t|-|t|_{\text {min }}$ constituted of the squared four-momentum transfer $t=-Q^{2}$ from the beam to the final state system, and $|t|_{\min }$ the minimum value of $|t|$ allowed by kinematics. The quasi-real photon density provided by a heavy nucleus of charge $Z$ is given by Weizsäcker and Williams [3]. It relates the experimentally observed cross-section of pion-nucleus scattering to $\pi \gamma$ scattering via

$$
\frac{d \sigma_{\pi-\text { nucleus }}}{d s d t^{\prime} d \Phi_{n}}=\frac{\alpha \cdot Z^{2}}{\pi\left(s-m_{\pi}^{2}\right)} \cdot \frac{t^{\prime} \cdot F_{\text {eff }}^{2}\left(t^{\prime}\right)}{\left(t^{\prime}+t_{\min }\right)^{2}} \cdot \frac{d \sigma_{\pi \gamma}}{d \Phi_{n}}
$$

with $\sqrt{s}=m_{X}$ the invariant mass of the final state system $X, d \Phi_{n}$ the phase-space element for this $n$-particle final state system, and $F_{\text {eff }}^{2}\left(t^{\prime}\right)$ the effective electro-magnetic form factor of the heavy nucleus.

The various final states potentially emerging from $\pi^{-} \gamma$ scattering can be used to investigate various topics, which are accessible by the COMPASS experiment (as well as the corresponding kaon induced $K^{-} \gamma$ reactions). They are summarized here without claiming completeness. Most prominently, the $\pi^{-} \gamma$ final state gives access to the pion polarizability, as discussed in section 2 . The $\pi^{-} \pi^{0}$ final state can be used to extract the chiral anomaly $F_{3 \pi}$. For three-pion final states, i.e. both $\pi^{-} \pi^{0} \pi^{0}$ and $\pi^{-} \pi^{-} \pi^{+}$, the calculation of the absolute cross-section has been provided by ChPT. The measurement of the charged case is given in section 3 as published in [2].

COMPASS is a multi-purpose fixed-target experiment at the SPS at CERN, that investigates the structure and spectroscopy of hadrons. COMPASS features a two-stage magnetic spectrometer [1] with high precision tracking detectors, particle identification and calorimetry, that can detect both charged and neutral outgoing particles within a wide range of scattering angles and particle momenta. Thereby it provides a uniform acceptance, especially for reactions featuring low to intermediate momentum transfer from the beam to the target.

In short periods in 2004 and 2009, data was taken with $190 \mathrm{GeV} / c$ hadron beam (composed of $98.6 \% \pi^{-}, 2.4 \% K^{-}, 0.8 \% \bar{p}$ ) impinging on thin lead or nickel disk targets, respectively. In 
both cases, additional silicon micro-strip detectors were installed downstream of the target, for optimized vertexing even for smallest scattering angles, which are characteristic for Primakoff reactions, and also for other reactions at low momentum transfer. The relevant components of the trigger were a multiplicity trigger for events with several charged particles in the final state, and a trigger based on energy deposits in the electromagnetic calorimeter (ECAL) for final states with neutral particles.

\section{Pion Polarizabilities}

In the classical picture, electric and magnetic dipole moments as consequences of the action of an external electro-magnetic field are characterized by the electric polarizability $\alpha$ and the magnetic polarizability $\beta$. For pions, the electric and magnetic polarizabilities $\alpha_{\pi}$ and $\beta_{\pi}$ characterize the behaviour of the quark-gluon structure, i.e. the deformation of the pion, in an electro-magnetic field. Thus calculations within various theoretical models have been carried out, with different results of these parameters. The existing experimental results vary on even larger scales, so that a measurement with higher precision is desired since long.

The pion polarizabilities can be experimentally studied in Primakoff Compton scattering, where the deformation of the pion in the electro-magnetic field of the quasi-real photon leads to the emission of another real photon. The leading deviation of the Compton cross-section, depending on both the scattering angle $\theta_{c m}$ of the $\pi \gamma$ system and its invariant mass $\sqrt{s}$, from the cross-section of the Compton scattering of a point-like particle, gives access at leading and next-to-leading order to $\alpha_{\pi}+\beta_{\pi}, \alpha_{\pi}-\beta_{\pi}$, and the quadrupole polarizability combination $\alpha_{2}-\beta_{2}$ :

$$
\frac{d \sigma_{\pi \gamma}}{d \Omega_{c m}}=\frac{\alpha^{2}\left(s^{2} z_{+}^{2}+m_{\pi}^{4} z_{-}^{2}\right)}{s\left(s z_{+}+m_{\pi}^{2} z_{-}\right)^{2}}-\frac{\alpha m_{\pi}^{3}\left(s-m_{\pi}^{2}\right)^{2}}{4 s^{2}\left(s z_{+}+m_{\pi}^{2} z_{-}\right)} \cdot \mathscr{P}
$$

with

$$
\mathscr{P}=z_{-}^{2}\left(\alpha_{\pi}-\beta_{\pi}\right)+\frac{s^{2}}{m_{\pi}^{4}} z_{+}^{2}\left(\alpha_{\pi}+\beta_{\pi}\right)-\frac{\left(s-m_{\pi}^{2}\right)^{2}}{24 s} z_{-}^{3}\left(\alpha_{2}-\beta_{2}\right)
$$

where $z_{ \pm}=1 \pm \cos \theta_{c m}, m_{\pi}$ the pion mass, and $\alpha$ the fine structure constant. The cross-section given by equation 2.1 is most strongly influenced by $\alpha_{\pi}+\beta_{\pi}$ in forward scattering, and by $\alpha_{\pi}-\beta_{\pi}$ in backwards scattering in the center-of-mass system. The comparison to the cross-section of the Compton scattering on a point-like particle has to be carried out by comparison of the experimental data to a dedicated Monte Carlo simulation of a point-like pion scattering off the nuclear target in the respective spectrometer.

The COMPASS 2009 data with ECAL trigger, dedicated to the measurement of the pion polarizabilities, is currently being analyzed. A set of 23000 exclusive $\pi^{-} \gamma \rightarrow \pi^{-} \gamma$ events fulfill the kinematic cuts on the reconstructed vertex, beam particle identification, photon energy, small $Q^{2}$, small invariant mass of the $\pi^{-} \gamma$ system, minimum transverse momentum of $50 \mathrm{MeV} / c$ of the scattered pion, needed to identify the interaction point in the target, and the fraction of the photon energy w.r.t. the beam energy above $50 \%$. This data set serves as a basis for the extraction of the polarizability difference $\alpha_{\pi}-\beta_{\pi}$ under the assumption $\alpha_{\pi}+\beta_{\pi} \approx 0$. Figure 1 (left) shows the energy balance of these $\pi^{-} \gamma$ final state events. The width of the exclusivity peak is $\sigma \approx 2.6 \mathrm{GeV}$ and in good agreement for data and simulation. Figure 1 (right) shows the $Q^{2}$ spectrum where the 

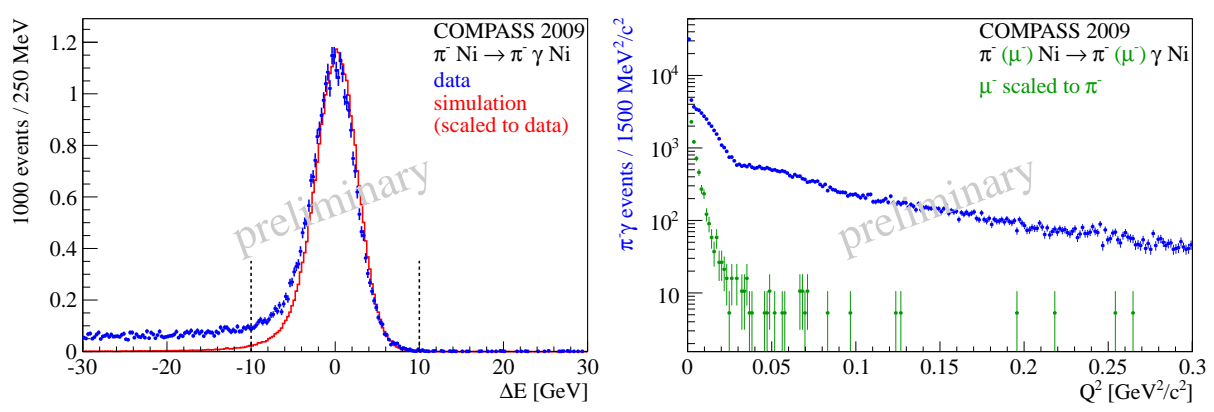

Figure 1: Polarizability data: Energy balance of the $\pi^{-} \gamma$ final states, with the exclusivity cut indicated (left). $Q^{2}$ spectrum for both pion and muon measurement with the typical Primakoff peak in the first bin (right).

Primakoff peak lies in the first bin. A cut on $Q^{2}<0.0015 \mathrm{GeV}^{2} / c^{2}$ is applied for the analysis to separate the photon exchange from strong interaction. This figure includes also the $Q^{2}$ spectrum from the muon control measurement, normalized in the first bin to the pion data. This features both a pure electro-magnetic interaction, and a point-like beam particle without any polarizability effects. This muon control measurement is a unique feature of the COMPASS setup, and as such provides a rigorous check of the spectrometer description in the simulation.

The expected uncertainty for $\alpha_{\pi}-\beta_{\pi}$ from this data is better than $1 \cdot 10^{-4} \mathrm{fm}^{3}$.

\section{Chiral Dynamics in $\pi^{-} \gamma \rightarrow \pi^{-} \pi^{-} \pi^{+}$}

The cross-sections for $\pi \gamma \rightarrow 3 \pi$ reactions at low energies have been calculated using ChPT at leading order [4], and including loops and counter-terms [5]. They are experimentally accessible in Primakoff reactions, as depicted for the possible tree diagrams in figure 2.

In experimental $\pi^{-} \mathrm{Pb} \rightarrow \pi^{-} \pi^{-} \pi^{+} \mathrm{Pb}$ data, diffractive production via Reggeon $t$-channel exchange prevails, while the Primakoff production of the same final state has its dominant contribution at $t^{\prime} \approx 0$. Primakoff production introduces spin projection $M=1$ to the produced system, while diffractive production features $M=0$ at vanishing momentum transfer. As the low-mass region, i.e. $m_{3 \pi}<0.72 \mathrm{GeV} / c^{2}$, is governed by pion scattering instead of resonance production, the ChPT calculations are supposed to be applicable for the relevant Primakoff-produced events. As both
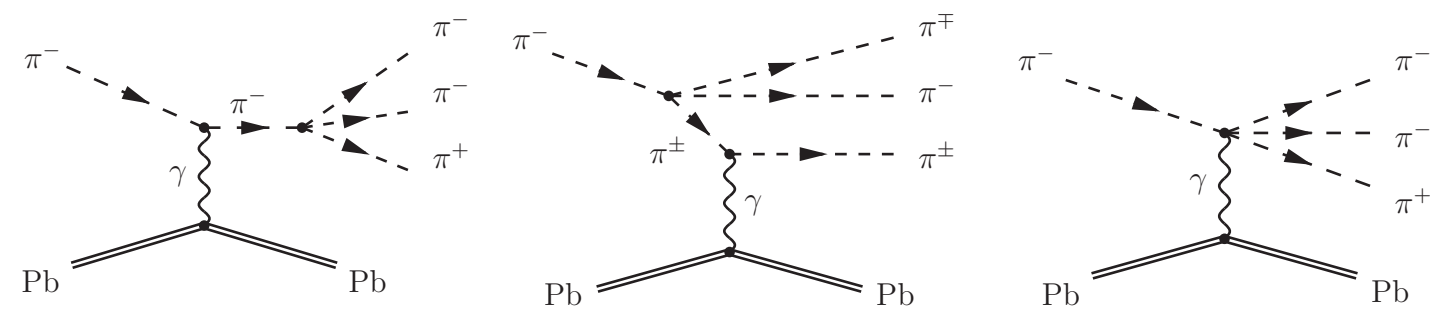

Figure 2: Leading order processes in ChPT [4] for the reaction $\pi^{-} \gamma \rightarrow \pi^{-} \pi^{-} \pi^{+}$, embedded in the Primakoff reaction contribution to $\pi^{-} \mathrm{Pb} \rightarrow \pi^{-} \pi^{-} \pi^{+} \mathrm{Pb}$. 

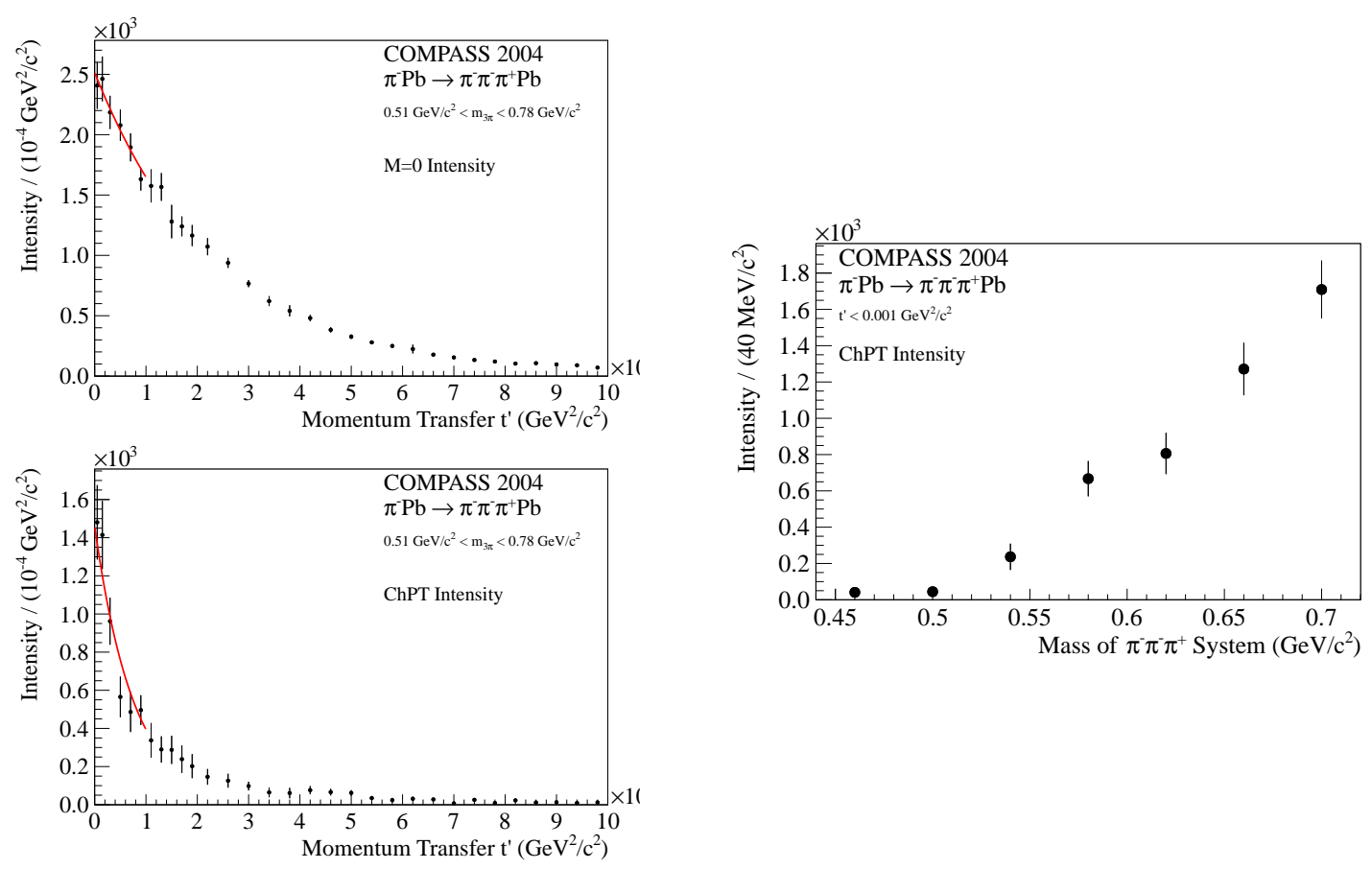

Figure 3: $\pi^{-} \pi^{-} \pi^{+}$data: The $t^{\prime}$ dependence of the $M=0$ amplitudes (left top) illustrates their diffractive production. The $t^{\prime}$ dependence of the chiral amplitude (left bottom) demonstrates Primakoff production. The total intensity of the chiral amplitude (right) is converted to the absolute cross-section, which is compatible with the ChPT calculations [2].

calculated cross-sections, i.e. with and without loops, are almost identical up to about $\sqrt{s}=5 m_{\pi}$, it is sufficient to restrict to the leading-order calculations for the first comparison with experimental data in the low-mass range.

The contribution of the processes calculated by ChPT to an experimental data set can be examined by the techniques of partial-wave analysis (PWA). The traditional goal of a PWA is the determination of all resonances present in a given data set, and their properties, by fitting angular distributions and taking into account interference effects, but without pre-defining relative strenghts. This approach may be extended by considering also non-resonant contributions, if their angular relations are established. The PWA presented in the following is still based on the commonly-known isobar model, that assumes sequential decays of resonances via intermediate two-particle decays into the particles observed in the spectrometer. For $3 \pi$ events, a partial wave in the reflectivity basis is written as $J^{P C} M^{\varepsilon}[i$ isobar $\pi] L$, defining the quantum numbers of the resonance, $J^{P C}$, spin projection $M$, reflectivity $\varepsilon$, the isobar, and the angular momentum $L$ between the di-pion resonance and the unpaired pion. This model has been extended by a chiral amplitude, that consists of the fully differential form of the calculations from [4].

The COMPASS 2004 data, taken with multiplicity trigger and lead target, provides about 4 million exclusive $\pi^{-} \mathrm{Pb} \rightarrow \pi^{-} \pi^{-} \pi^{+} \mathrm{Pb}$ events. Out of those, $\sim 1$ million feature $t^{\prime}<0.001 \mathrm{GeV}^{2} / c^{2}$, which is the kinematic region with presumably Primakoff production in the $M=1$ contribution. These data are subject to the PWA, in a way, that used the chiral amplitude to describe pure pion scattering processes including a photon. The chiral amplitude is mathematically not orthogonal to 
the isobaric $M=1$ amplitudes, so that these have in fact to be replaced by the chiral amplitude in the low-mass region. A dedicated PWA fit in bins of $t^{\prime}$, covering the low-mass region, showed that the dependence on $t^{\prime}$ is indeed consistent with Primakoff production of the chiral amplitude, while the competing $M=0$ contributions stem from diffractive production (see figure 3, left plots). For the comparison with the ChPT calculations, a PWA fit has been carried out in $40 \mathrm{MeV} / c^{2}$ mass bins in the mass region $0.44<m_{3 \pi}<0.72 \mathrm{GeV} / c^{2}$. The resulting fit quality is compatible with the quality of a previous fit without the chiral amplitude, that had used up to 6 isobaric waves instead, but the new fit works with much less fitting parameters. In addition, the use of these 6 isobaric waves had been of pure empirical nature, and there is no physical motivation for those to appear. The fitted total intensity of the chiral amplitude, shown in the right plot of figure 3 , is normalized by the incoming beam flux, which is obtained from the decay of beam kaons into the same final state, and the target thickness. The obtained cross-section for $\pi \mathrm{Pb}$ scattering needs then to be divided by the quasi-real photon density as given by equation 1.1. The resulting cross-section for $\pi^{-} \gamma \rightarrow \pi^{-} \pi^{-} \pi^{+}$is measured with a total uncertainty of $20 \%$ and, on this level, in good agreement with the calculations from ChPT [2].

\section{Conclusion and Outlook}

The results presented in this paper summarize measurements at COMPASS related to ChPT calculations.

The 2009 polarizability data will serve for a measurement of $\alpha_{\pi}-\beta_{\pi}$. With the 2012 high statistics polarizability data, even the separation of $\alpha_{\pi}$ and $\beta_{\pi}$ is expected to be possible.

The first measurement of the $\pi^{-} \gamma \rightarrow \pi^{-} \pi^{-} \pi^{+}$absolute cross-section in the low-mass region, from 2004 data, is compatible with the leading-order ChPT calculations. The partner reaction $\pi^{-} \gamma \rightarrow \pi^{-} \pi^{0} \pi^{0}$, contained in the 2009 Primakoff data, is expected to give a first insight into the necessity of loop diagrams in this mass region. For both analyses, higher-order contributions, i.e. loops and explicit rho contributions, have to be taken into account for a proper analysis of the adjacent region of higher masses. The $3 \pi$ Primakoff data still have further physics potential: The radiative excitation of resonances like the $a_{2}(1320)$ can reveal their radiative width, and also the interference of Primakoff and diffractive production of these resonances can be investigated.

\section{References}

[1] COMPASS collaboration, P. Abbon et al., The COMPASS Experiment at CERN, Nucl. Instrum. Meth. A577, 455 (2007) [hep-ex/ 0703049 ].

[2] COMPASS collaboration, C. Adolph et al., First Measurement of Chiral Dynamics in $\pi^{-} \gamma \rightarrow \pi^{-} \pi^{-} \pi^{+}$, Phys. Rev. Lett. 108, 192001 (2012) [hep-ex/1111.5954].

[3] I. Ya. Pomeranchuk and I. M. Shmushkevich, On Processes in the Interaction of $\gamma$-Quanta with Unstable Particles, Nucl. Phys. 23, 452 (1961).

[4] N. Kaiser and J. Friedrich, Cross-sections for low energy $\pi^{-} \gamma$ reactions, Eur. Phys. J. A36, 181 (2008) [nucl-th/0803.0995].

[5] N. Kaiser, Chiral corrections to $\pi^{-} \gamma \rightarrow 3 \pi$ processes at low energies, Nucl. Phys. A848, 198 (2010) [hep-ph/1007.5277]. 